\title{
Problèmes posés par l'adaptation des ouvrages à la nature et au relief des fonds marins
}

Lexploitation des champs pétroliers, particulièrement en mer du Nord, a suscité la construction d'ouvrages nouveaux : vastes caissons de béton armé el précontraint posés au fond et qui tirent de leur poids leur stabilité.

Le premier ouvrage réalisé à Ekofisk sera suivi cet été par la mise en place de deux structures gravitaires d'un type différent: en plan, la surface d'appui au sol est de l'ordre de l'hectare.

En dehors du poids. les forces prépondérantes sont déterminées par laction des houles de tempete; pour la vague centenaire en mer du Nord. on considere des forces horizontales de 25 à $40000 t$ et des moments de renversement de 1 à 2 millions de $t / m$.

Seuls des sols assez fermes pour subir des contraintes importantes. malgré les affouillements inévitables, permettent l'emploi de telle structure: les fonds du golfe du Mexique, berceau de l'exploitation offshore, ne répondent pas a ces caractéristiques; la mer du Nord au contraire a révélé des fonds assez raides.

Je voudrais ici passer en revue les difficiles problèmes liés à l'hydraulique maritime que soulevent ces constructions:

- la forme des fonds:

- le comportement de l'eau contenue dans le sol sous l'effet des forces alternées dues a la houle:

- la tenue des fonds après la pose:

- la mise en place des caissons.

N'attendez pas de cet exposé des solutions.

\section{Nature et forme des fonds}

Les fonds de la mer du Nord sont constitués de couches alternées, soit d'origine glaciaire, ou surchargées par d'an- ciens glaciers. soit par des dépôts marins: durant le quatemaire, le niveau des mers a souvent varié mettant jusqu à sec la mer du Nord qu'occupaient alors des glaciers venant surtout de Scandinavie.

Des couches consolidées par d'importantes charges de glace ont été creusées de talweg sous les glaciers. puis la mer a repris son empire et des matériaux fins ou sableux ont eté lransportés par les actions marines. masquant certains des reliefs anciens: ce processus sest répété à plusieurs reprises.

Des dunes. des rides diverses se rencontrent au fond: mais dans létat actuel de nos connaissances sur l'action de la houle sur le fond. que peut-on en dire?

La communication de M. Cavanié, qui va suivre. examine les courants de houle au voisinage du fond: la même étude pourrait être fate pour la mer du Vord.

Les résultats seraient voisins: ce n'est que pour une wes fable fraction du temps total que la houle atteint une amplitude suffisante pour remanier vraiment les matériaux du fond. mais à l'échelle des temps géologiques cela comple.

Des études sur leffet de la variation de pression au fond sur les couches profondes. jusqu $\ddot{a} 200 \mathrm{~m}$ sur le fond, montrent que ces pulsations répétées déterminent des cisaillements suffisamment importants pour tasser les couches sableuses. mais sont sans action sur les couches argileuses.

Nous reviendrons plus longuement sur ce point : ce qui est sûr. cest que la variation de pression de leau au fond est trop rapide pour pouvoir se transmettre a leau interstilielle. la variation de pression de leau agit sur le sol luimême. sauf peut-être pour les couches très proches de la. sulface.

Lorsque les couches argileuses sont denses. c'est qu elles ont été compactées par le poids du ou des glaciers qui les ont surmontées.

On peut donc rencontrer une succession de couches de sable et d'argile. toutes compactes, mais aussi des couches 
argileuses peu denses comprises entre deux couches de sable dense.

Ces remarques concernent les cent à cent cinquante premiers mètres de fond qui interviennent assez directement dans la stabilité des embases dont la plus petite dimension est de cet ordre de grandeur.

Une meilleure connaissance de ce mécanisme daction des houles sur le fond est tout à fait nécessaire.

On souhaiterait aussi que la zone superficielle sujette au modelage de la houle soit assez plastique pour épouser la forme de la base du caisson: aussi une bonne connaissance du mécanisme de formation des dunes et rides, de la répartition des différentes granulométries des matériaux et de leur densité permettrait de pallier une certaine insuffisance dans la reconnaissance des fonds. reconnaissance longue, difficile et coûteuse surtout pour les couches meubles de la surface.

\section{Tenue des fonds après la pose du caisson}

Nous rencontrons ici deux problèmes:

- l'un fait partie de la mécanique des sols mais met en jeu le comportement de l'eau dans le sol;

- l'autre relatif à l'hydraulique maritime concerne l'érosion des fonds autour d'une structure.

\section{COMPORTEMENT DU SOL SOUS L'EFFET DES CONTRAINTES ALTERNÉES}

L'effet des houles se traduit par des cisaillements dans le sol qui varient sensiblement sinusoïdalement dans le temps, et par des variations de contraintes verticales qui oscillent autour d'une valeur moyenne.

Ces contraintes sont équilibrées pour partie par les forces de contact entre les grains du sol, pour partie par leau contenue dans le sol.

Dans le cas des sols sableux, une action plus forte que celle antérieurement subie est susceptible de modifier la position relative des grains et donc de déterminer une réduction du volume occupé. Cette réduction de volume n'est effective que si une certaine quantité d'eau arrive à être expulsée : je me place dans l'hypothèse d'un sol saturé.

Les forces de cisaillement provoquées par la houle agissant sur les caissons sont plus grandes que les forces dues à la houle seule avant tous travaux.

L'action de la houle va donc induire un réarrangement des grains, mais la tempête est trop courte pour que l'eau interstitielle ait le temps de s'évacuer, et de toute façon ne s'évacuera que s'il existe un gradient de pression. Différentes études de laboratoire, reprenant celles développées antérieurement pour l'étude de la liquéfaction des sols pendant les séismes, ont laissé entendre que, lors d'une tempête, la pression interstitielle, c'est-à-dire l'excès de la pression de leau dans le sol par rapport à la pression hydrostatique, croissait constamment. Or, dans un sol sableux, la résistance au cisaillement dépend seulement des contraintes intergranulaires: ici, la contrainte totale est constante; si la pression interstitielle croit, c'est que la contrainte intergranulaire décroit et avec elle la résistance au cisaillement.

Après la tempête, la pression interstitielle se dissipe par suite de la perméabilité du sol et, au final, le sol se trouve dans un état de plus grande densité donc apte à résister à une tempête plus forte.

Étudier la stabilité d'une plateforme c'est donc apprécier les risques de liquéfaction du sol au cours des premières tempêtes.

Cette approche, qui peut paraitre théorique, a reçu une confirmation par les mesures effectuées en novembre 1973 sur le caisson d'Ekofisk, le caisson ayant été mis en place au cours de l'été 1973.

Le 6 novembre, pour des houles d'amplitudes significatives de $11 \mathrm{~m}$ environ, on a mesuré dans les sables sousjacents des montées de la pression interstitielle de 2 à $4 \mathrm{t} / \mathrm{m}^{2}$, et jusqu'à $6 \mathrm{t} / \mathrm{m}^{2}$ dans une couche argileuse.

Dans les jours qui ont suivi la tempête, la plateforme a tassé d'un centimètre, le tassement au cours de la tempète ayant atteint deux centimètres.

C'est par un processus analogue que la houle agissant sur le fond de la mer est susceptible de compacter les sols sableux: tout dans le phénomène n'est pas encore bien clair et le comportement des sols argileux reste encore très mal connu.

\section{ÉROSION}

L'étude de l'érosion des fonds, sous l'action de la houle agissant autour d'obstacles, est assez ancienne; on a appliqué aux nouvelles structures, lors des essais en bassin, les conditions de similitude assez bien dégagées antérieurement.

Rappelons qu'il faut reproduire la vitesse de début d'entrainement sans employer des grains d'une taille telle qu'elle excède l'épaisseur de la couche limite. Pour des houles de 15 à 20 secondes de période, l'épaisseur de la couche limite est de l'ordre de 5 à $10 \mathrm{~mm}$; les grains de sable qui tapissent une bonne part de la mer du Nord ont $0.2 \mathrm{~mm}$ de $d_{50}$ et exceptionnellement 0,6 à Frigg.

Une faible variation de la période a des répercussions importantes sur la cinématique de la houle : c'est ainsi que pour la vague centenaire en mer du Nord, de $30 \mathrm{~m}$ d'amplitude, on a en utilisant la théorie de la houle au premier ordre :

\begin{tabular}{|l|c|c|c|}
\hline Période $\ldots \ldots \ldots \ldots \ldots \ldots .(\mathrm{s})$ & 16 & 18 & 20 \\
\hline Vitesse maximale au fond $(\mathrm{m} / \mathrm{s})$ & 1,06 & 1,53 & 1,81 \\
\hline Excursion totale au fond $\ldots(\mathrm{m})$ & 5,4 & 8,8 & 11,5 \\
\hline
\end{tabular}

Il faut penser que, dans l'état actuel de nos connaissances, lamplitude des houles extrêmes est connue de façon plus sûre que la période.

Cependant, la question des érosions ne parait pas très aiguë : on a constaté des fosses d'érosion au voisinage des angles et de toutes les singularités des caissons. La remarque faite par $M$. Larras antérieurement, que pour une durée d'action infinie la profondeur et la forme des fosses étaient indépendantes de la granulométrie des matériaux et 
dépendatent de la densité des grains. a été en général vérifiée : elle permet de simplifier le processus dessai.

La présence d'un caisson tend en général à modifier la forme des rides et crée des structures de rides souvent très esthétiques, quelquefois une modulation à léchelle du caison intervient pour onduler la surface moyenne sur laquelle se placent les dunes.

On constate aussi en bassin que si la structure s'appuie sur le fond. sans écraser toutes les rides, il se produit, en raison des déphasages dans le champ de pression au fond. des circulations d'eau importantes dans les creux des rides entrainant des affouillements internes: les conditions de similitude de mécanique des sols ne sont pas respectées et il n'est pas sur que cette situation puisse se rencontrer en nature.

\section{La mise en place des caissons}

Il faut rappeler à nouveau que poser au fond de la mer une structure dont la base couvre 1 ha et est sensiblement plane suppose un fond de la mer très plat et somme toute régulier.

Lors de limmersion du caisson. il arrive que le volume d'eau compris entre le fond et la base du caisson se réduise à une couche d'épaisseur faible : c'est un peu l'équivalent dun palier Mitchell de turbine. Dès lors qu'il existe un petit angle entre le fond et le radier. le mouvement descendant du caisson crée une force horizontale très importante couplée à une réaction verticale ascendante. Le systeme de positionnement au moment de la pose doit tenir comple de ces efforts. Mais on dispose encore d'assez peu de résultats pour préciser ce phénomène.
Lorsque le caisson a touché le fond, s'il est muni de bêches sur sa périphérie, il convient d'assurer une bonne pénétration des bêches dans le sol, combinée à l'évacuation de leau excédentaire contenue entre le radier du caisson, les bêches, le sol.

Il importe que cette vidange ne se fasse pas par passage sous les bêches, car il en résulterait des érosions et par suite une non continuité du contact des bêches et du sol d'appui. objectif tout à fait opposé à celui poursuivi par la construction des bêches. Un dispositif hydraulique adéquat est done nécessaire, dont le fonctionnement est lié à la pénétration des bêches: autre exemple d'un problème à la jonction de l'hydraulique et de la mécanique des sols

\section{Conclusion}

La réalisation des structures gravitaires posées au fond de la mer du Nord a ouvert de nouveaux chapitres dans les traités d`Hydraulique Maritime et de Mécanique des Sols.

Jaai évoqué ici les principaux sujets de recherches abordés jusqu'ici; on peut penser que la liste n'est pas exhaustive.

Je voudrais, pour terminer, remercier la Société Hydrotechnique de France d'avoir ouvert un débat sur ces nouveaux sujets et émettre le souhait, qu'au sein de cette société savante, des efforts soient entrepris pour améliorer nos connaissances fondamentales de l'interaction entre fond et houle; voie prometteuse pour appronfondir ce qui touche au sol et à la houle elle-même; vous savez tous combien lampleur des houles au large, repérée de façon sûre depuis peu, a surpris, les données de la houle à la côte ne laissant pas prévoir de si fortes amplitudes.

\section{Discussion}

Président: M. le Professeur LACOMBE

M. le Président remercie M. COUPRIE pour son mémoire particulièrement intéressant, non seulement du fait du sujet traité mais encore par le caractère pluridisciplinaire de son exposé. Trop souvent, les spécialistes se désintéressent de ce qui se passe à l'interface de deux milieux, mais le moment est venu de se préoccuper de l'interface "eau-fond marin ". Il ouvre ensuite la discussion.

Comment ont été mesurées les pressions sous le réservoir sous-marin d'Ekofisk? demande M. LAvGunLLET (Sté Technip)

Lorsque le caisson d'Ekofisk a été mis en place, répond $M$. COUPRIE, diverses "réservations", faites à cet effet, ont permis de forer au travers du caisson et dans le sol un certain nombre de trous dans lesquels des piézomètres ont été mis en place et scellés. Chaque piézomètre comporte à sa base deux petites cellules de captation, lune avec une sorte de corde vibrante, l'autre plus classique. Il y a, au total, 28 sondes pour la mesure des pressions interstitielles sous le caisson. Le mieux serait de vous reporter a la communication faite sur ce sujet en mai dernier au Congrès de I'O.T.C. à Houston (off shore Technical Conference).

M. Sommer voudrait savoir si ces piézomètres ont permis de détecter sous le caisson des variations de pression dues à la houle.
Il y a tant d'appareils enregistreurs sur le caisson, dit $M$. CouPRIE, qu'on n'enregistre que les maxima horaires des pressions et non une courbe continue des variations de celles-ci. L'ouvrage a essuyé deux tempêtes mais, pour des raisons financières, les mesures n'ont pas été effectuées au cours de la dernière. De toute manière, il y a beaucoup de renseignements qui ne sont pas encore complètement exploités.

Sur une question de $\mathrm{M}$. le Président, M. COUPrIE indique que la mesure de l'enfoncement dans le sol du réservoir d'Ekofisk a été relativement aisée. Il existe, en effet, à environ $100 \mathrm{~m}$ de l'ouvrage, une structure fondée sur des pieux battus dans le sol à une profondeur de $100 \mathrm{~m}$; on a considéré cette structure comme un point fixe. Par contre, il n'a pas été possible, semble-t-il, de mesurer les déplacements latéraux du réservoir au cours des tempêtes.

Vous avez souligné. remarque $M$. SPARKs, que la liquéfaction du sol tend à croître après une tempéte. A-t-on pu vérifier la montée des pressions intersticielles sous Ekofisk I pendant une deuxième tempête après celle de novembre 1973 ?

M. COUPRIE répond :

En novembre 1973, il y a eu deux tempêtes très fortes. La première a provoqué déjà un certain tassement; à la deuxième 
tempête, le tassement a été beaucoup plus important : Ekofisk, depuis qu'il est en place, s'est tassé de $5 \mathrm{~cm}$. L'hiver dernier, les tempêtes n'ont pas été aussi fortes.

Les tempêtes du premier hiver semblent être des tempêtes décennales. Il peut arriver que la tempête décennale se produise au cours du premier hiver; mais cela veut dire qu'il va peut-être falloir attendre cinq ou six ans avant d'avoir une tempête d'un bon niveau pour faire une nouvelle série de mesures intéressante.

M. BEllamy pose les deux questions ci-après :

$1^{\circ}$ Pensez-vous qu'une variation des contraintes normales sur le sol puisse ètre préjudiciable à la tenue des sols? En particulier l'annulation de ces contraintes normales sur les sables pourraitelle conduire à une liquéfaction de ceux-ci?

$2^{\circ} \mathrm{A}-\mathrm{t}$-on constaté des affouillements à Ekofisk?

Sur le premier point, répond $M$. Couprie, je dirai que les plateformes sont dimensionnées pour qu'on n'en vienne pas à l'annulation des contraintes normales.

En ce qui concerne le second, à ma connaissance, on n'a pas constaté d'affouillement mais il est assez difficile de mesurer de combien le caisson s'enfonce par rapport au sol. D'ailleurs, les caissons Jarlan, employés ici, sont réputés, à juste titre, pour réduire les effets d'érosion au voisinage du caisson; en particulier le courant d'échange, créé lors de la vidange du réservoir, est assez efficace pour réduire à une valeur de sécurité les courants au voisinage du fond dans la région considérée.
Sur une question de M. le Président, M. Couprie confirme la difficulté d'une exploration initiale du sol et surtout du sous-sol de fondation. Ce dernier devrait être connu sur une hauteur égale à la plus grande dimension de la structure car le champ des contraintes est encore important à cette profondeur. Or, par suite de difficultés de carottage notamment, on est loin de connaître, ici. les caractéristiques mécaniques du terrain de fondation avec la même précision que dans les fondations de grands ouvrages sur la terre ferme.

Je peux préciser, dit M. Montargès, que le seul procédé de reconnaissance actuellement utilisé en mer du Nord est le procédé de forage avec carottage au cáble par percussion. Lat séquence de prise de carottes est, au debut, dune carotte tous les 3 pieds, mais, à partir d'une trentaine de mètres, on se contente d'une carotte tous les 10 pieds et même davantage. De plus, pendant la prise de carottes, on arrête la circulation de boue, ce qui provoque des dépôts de sédiments en suspension dans la boue et fausse les résultats déduits des carottes. On utilise de plus un pénétromètre - le pénétromètre Sea Calf - dont la pénétration maximale est de $24 \mathrm{~m}$.

En l'absence d'autre intervention, M. le Président clôt la discussion en remerciant tous ceux qui ont contribué à l'animer et tout particulierement M. COUPRIE.

Il donne ensuite la parole à M. R. Ezraty pour l'exposé de la communication qu'il a établie en collaboration avec M. ARHAN. 